\section{Author Reply: Re: Axillary Staging in the Neoadjuvant Setting}

\section{TO THE EDITORS:}

We appreciate the comments by Olsha and Carmon regarding timing of axillary lymph node dissection (ALND) in patients with a positive sentinel lymph node (SLN) undergoing neoadjuvant chemotherapy (NAC). In our review of axillary staging in the neoadjuvant setting we recommend performing ALND with the definitive breast surgery after completion of NAC in those identified as SLN-positive prior to NAC. ${ }^{1}$ The primary concern of the authors is that information regarding the remaining axillary nodes may be lost by delaying completion ALND until after the NAC is completed. They feel loss of this information may result in change in recommendations regarding lymphatic basin radiation or chest wall radiation in those undergoing mastectomy. A secondary concern of the authors is that delay of ALND requires reoperation of the axillary basin, which because of fibrosis and scarring may be more technically difficult than performing ALND in a field where there has been no prior surgery. They recommend performing frozen section at the time of SNB and completing the ALND at the same time if the sentinel node harbors metastases. This is, of course, a reasonable option.

We agree that the nodal status of the nonsentinel nodes may be altered by NAC, potentially leading to downstaging of the axilla. The post-NAC status of the nonsentinel nodes may change the recommendations for lymphatic basin radiation. However, in the neoadjuvant setting, recommendations for nodal or postmastectomy radiation are often based on primary tumor size. Frequently, those who present initially with clinically palpable disease in the axilla and whose nonsentinel nodes have been downstaged by NAC would still be likely to receive third-field radiation. There is evidence to support third-field radiation even for small tumors in premenopausal women with lymph node involvement. ${ }^{2-6}$ Reoperative axillary surgery after a SNB is usually not technically different and is facilitated by dissecting around the planes of previous dissection.
With increased use of preoperative axillary ultrasound and needle biopsy, many patients will be diagnosed with lymph node involvement without requiring SLN biopsy.

In conclusion, we endorse the authors' suggestion of performing ALND prior to NAC as an acceptable alternative pathway to axillary staging in patients undergoing NAC, acknowledging that there may be more ways than one to "skin a cat." The only potential disadvantage of their approach is that there may be a delay in initiation of chemotherapy.

\section{Alice Chung, MD, and Armando Giuliano, MD}

Department of Surgical Oncology, John Wayne Cancer Institute at Saint John's Health Center, Breast \& Endocrine, Santa Monica, CA

e-mail: alicepingchung@hotmail.com

Published Online: 1 January 2011

(C) Society of Surgical Oncology 2010

\section{REFERENCES}

1. Chung A, Giuliano A. Axillary staging in the neoadjuvant setting. Ann Surg Oncol. 2010;17:2401-10.

2. Truong PT, Olivotto IA, Kader HA, Panades M, Speers $\mathrm{CH}$, Berthelet E. Selecting breast cancer patients with T1-T2 tumors and one to three positive axillary nodes at high postmastectomy locoregional recurrence risk for adjuvant radiotherapy. Int $J$ Radiat Oncol Biol Phys. 2005;61:1337-47.

3. Overgaard M, Hansen PS, Overgaard J, Rose C, Andersson M, Bach F, et al., Postoperative radiotherapy in high-risk premenopausal women with breast cancer who receive adjuvant chemotherapy. Danish Breast Cancer Cooperative Group 82b Trial. N Engl J Med. 1997;337:949-55.

4. Ragaz J, Jackson SM, Le N, Plenderleith IH, Spinelli JJ, Basco $\mathrm{VE}$, et al. Adjuvant radiotherapy and chemotherapy in nodepositive premenopausal women with breast cancer. $N$ Engl J Med. 1997;337:956-62.

5. Recht A, Gray R, Davidson NE, Fowble BL, Solin LJ, Cummings FJ, et al. Locoregional failure 10 years after mastectomy and adjuvant chemotherapy with or without tamoxifen without irradiation: experience of the Eastern Cooperative Oncology Group. $J$ Clin Oncol. 1999;17:1689-700.

6. Wallgren A, Bonetti M, Gelber RD, Goldhirsch A, CastiglioneGertsch M, Holmberg SB, et al. Risk factors for locoregional recurrence among breast cancer patients: results from International Breast Cancer Study Group Trials I through VII. J Clin Oncol. 2003;21:1205-13. 